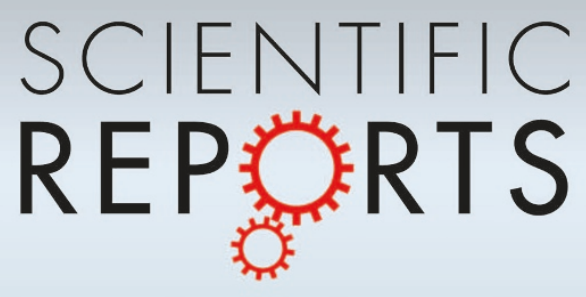

OPEN

SUBJECT AREAS:

NANOCAVITIES

NANOPHOTONICS AND

PLASMONICS

SUB-WAVELENGTH OPTICS

Received

10 October 2013

Accepted

29 November 2013

Published

9 January 2014

Correspondence and requests for materials should be addressed to

L.J.G. (guo@umich. edu)

\section{Transition from a spectrum filter to a polarizer in a metallic nano-slit array}

\author{
Jing Zhou \& L. Jay Guo
}

Department of Electrical Engineering and Computer Science, The University of Michigan, Ann Arbor, Michigan 48109, USA.

The transition from a spectrum filter (resonant transmission) to a polarizer (broadband transmission) for TM polarized light is observed in a metallic nano-slit array as period is decreased. A theoretical model is developed and shows that the spectrum filter behavior is caused by the coupled slit/grating resonance. With decreasing period, the slit resonance is decoupled from the grating resonance, which then dominates the transmission spectrum and broadens the transmission peak. With further reducing period, the slit resonance diminishes and the peak spectrum transforms to a broadband transmission. This effect is the basis for the operation of wire grid polarizers. The transition is explained by the change of the impedance to the incoming wave.

S ub-wavelength metallic nano-slit arrays have attracted much attention due to the extraordinary resonant transmission of transverse magnetic (TM) waves ${ }^{1-12}$. Based on the resonance behavior, metal slit arrays have been studied as polarizing spectrum filters ${ }^{13,14}$ and utilized in band-pass photodetectors ${ }^{15}$. Recently they become a promising candidate of the structural color filters in flat-panel displays and colored imaging sensors ${ }^{16,17}$. On the other hand, of a similar structure, metal wire grids have been extensively utilized as polarizers, with the characteristic high TM transmittance over broadband wavelength range ${ }^{18-23}$. A question naturally emerges: why does the similar kind of structures (metal slit arrays) have drastically different transmission characteristics? This work aims to reveal the mechanisms that are responsible for both effects. Especially we present experimental results and analysis to show the transition from a spectrum filter (resonant narrowband) to a polarizer (flat broadband) for TM waves in metal slit arrays.

A general observation is that the metal slit array based polarizers (or more commonly referred to as wire grid polarizer, WGP) usually have a smaller period than the spectrum filters. For example the typical period of a WGP working in the visible band is less than $150 \mathrm{~nm}^{20}$. Therefore, we study the evolution of the transmission spectrum of a metallic slit array as a function of its period. Experimentally we observed transition from a spectrum filter to a polarizer with decreasing period. In general there are two types of resonances associated with the structure: 1) the grating resonance, which is due to constructive interference of the scattered light by the periodic grating structure $^{24-27}$ and 2) the slit resonance from a localized Fabry-Perot resonance of the slit waveguide mode ${ }^{28-32}$. Many effects involve the interplay of both effects. Our theoretical analysis shows that the transition from resonant transmission in a spectrum filter to broadband transmission in a polarizer involves two processes: 1) decoupling of the slit resonance from the grating resonance and 2) diminishing of the slit resonance due to cancellation of induced polarization charges at the slit entrance. The slit resonance itself has a broad profile, which is confirmed by the transmission measurement of a slit array with very large period. Through the analogy between a metal slit array and a dielectric slab (following Ref. 33), an effective refractive index was derived which describes the diminishing process of the slit resonance, eventually giving rise to the broadband transmission of polarized TM light as in a typical WGP. In addition, we studied the angular dependence of the device as it changes from a spectrum filter to a polarizer and we found that the device becomes less angular dependent with decreasing period during the transition process.

\section{Results}

Decoupling of the coupled slit/grating resonance with decreasing period. Metallic nano-slit arrays are experimentally realized by creating perforating slits in an $\mathrm{Al}$ film on a fused silica substrate through standard e-beam lithography and reactive ion etching. Four periods $(d=200 \mathrm{~nm}, 300 \mathrm{~nm}, 400 \mathrm{~nm}$ and $500 \mathrm{~nm})$ with the same slit width $(a \sim 110 \mathrm{~nm})$ and the same slit height $(h \sim 170 \mathrm{~nm})$ are fabricated. The sketches and the SEM images of two samples with a period of $400 \mathrm{~nm}$ and a period of $200 \mathrm{~nm}$ are shown in Fig. 1. Firstly, the samples with four different periods are all polarization dependent. Typically both spectrum filters and polarizers require 


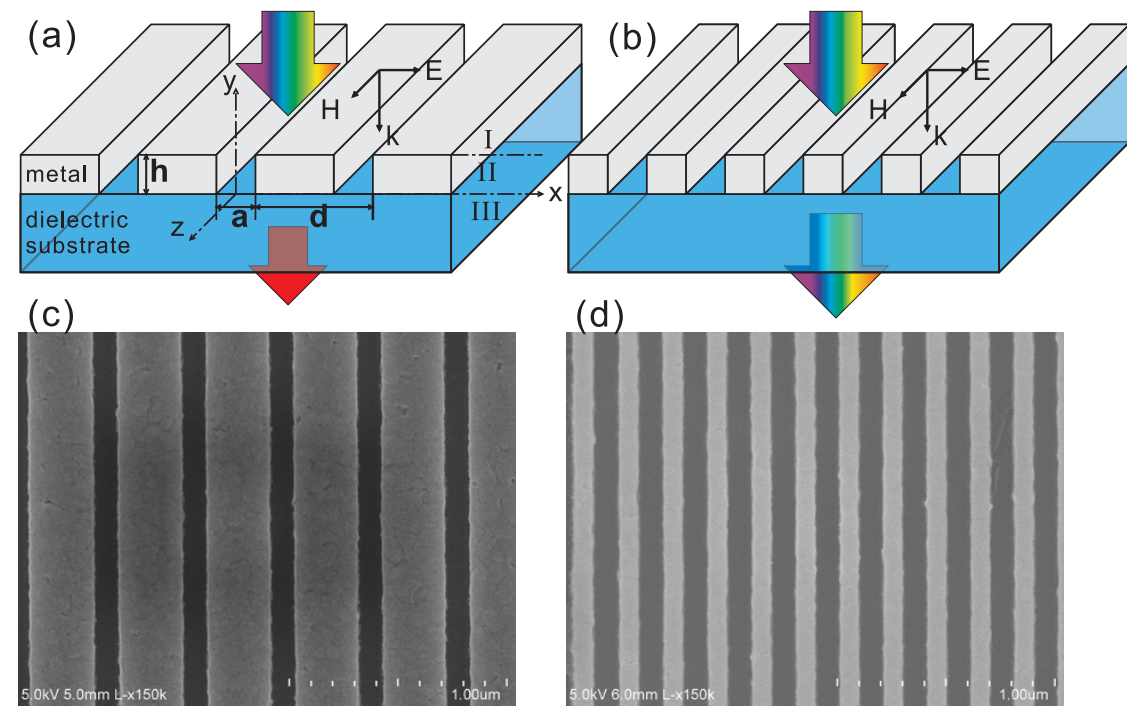

Figure $1 \mid$ Sketches and the corresponding SEM images of two metallic nano-slit arrays with different periods. (a) and (c) show a fused silica supported Aluminum slit array with a larger period $(400 \mathrm{~nm})$ serving as a spectrum filter. (b) and (d) show the similar structure with a smaller period (200 $\mathrm{nm}$ ) serving as a polarizer. Both these two structures have the same slit width of $\sim 110 \mathrm{~nm}$, and the same slit height of $\sim 170 \mathrm{~nm}$.

sub-wavelength scale metallic gratings, the incident TE wave is beyond the cut-off wavelength of the waveguide formed between the metal slit walls, therefore the TE waves are mostly reflected. So we focus on the behavior of TM polarized light. The transmission and reflection spectra together with photos of the four samples illuminated by a TM wave at normal incidence are shown in Fig. 2 (b) and (c), respectively. At a large period (e.g. $500 \mathrm{~nm}$ or $400 \mathrm{~nm}$ ), the resonant spectrum is prominent, giving rise to the distinctive transmission and complementary reflection colors. Resonant transmission peak or reflection dip is observed with asymmetric line shape. With decreasing period, the resonant peak or dip broadens and also shifts to shorter wavelengths. At the period of $200 \mathrm{~nm}$, the peak is so broad that the spectrum looks almost flat for the whole measurement range. Accordingly, a white transmission and a dark reflection for TM light are shown in the photos. In this experiment, the transition from a spectrum filter behavior (i.e. a resonant narrowband transmission) to a polarizer behavior (i.e. a broadband flat transmission) is clearly realized by simply varying the period of the metallic grating.

We used an analytical model based on mode expansion (see the Supplementary information online) to understand the observed effects. Besides the basic parameters of the slit array structure (such as slit width, slit height and period), the incident angle, the refractive index of the substrate and that of the slit filler can all be tuned in this model so that different measurements can be compared with theory by tuning the corresponding parameters in the model. Although the model assumes the metal to be a perfect conductor as an approximation, it captures most of the experimental features and provides a reasonable physical picture. Similar models have been established to describe the optical response of a grating ${ }^{1,2,5,25-27,29-31,34-36}$. We will discuss the effect of using real metal in the experiment later in this paper.

Due to the sub-wavelength scale of the slit array, the measured transmission is of the zeroth order (see the Methods section). At normal incidence and with air slits, the zeroth order transmission is derived by the model as

$$
T_{0}=\sqrt{\varepsilon_{d}}\left(\frac{a}{d}\right)^{2}\left|a^{-}-a^{+}\right|^{2},
$$

where $\varepsilon_{d}$ is the dielectric constant of the substrate; $a$ denotes the slit width; $d$ denotes the period; $a^{+}$and $a^{-}$are the coefficients of the fundamental slit waveguide modes propagating forwards and backwards, respectively. Following the experimental conditions, $\varepsilon_{d}$ is chosen to be 2.10 for a fused silica substrate; $a$ and $d$ are chosen to be $110 \mathrm{~nm}$ and $170 \mathrm{~nm}$, respectively. A 2D contour plot of the zeroth order transmission $T_{0}$ versus wavelength and period is shown in Fig. 2 (a). The red zone in the color map corresponds to the resonant transmission peak. The oblique dark lines (marked out by dashed white lines) represent the different diffraction orders of the grating resonances. They are divided into two sets due to the higher refractive index of the substrate than air. The two lines marked by $N^{\prime}=1$ and $N^{\prime}=2$ represent the first and the second order grating resonance at the grating/substrate interface while $N=1$ and $N=2$ represent the diffractions at the grating/air interface. The pronounced transmission resonance in the $2 \mathrm{D}$ contour plot is due to the coupling of the grating resonance with the slit resonance $e^{1,3-6,8,9}$. The slit resonance is a result of the standing wave formation due to the two counter-propagating waves represented by $a^{+}$and $a^{-}$, i.e. a Fabry-Perot resonance. Such slit resonance is independent of the period due to its localized characteristic. In addition, the slit resonance (marked by the band between the two vertical dash lines in Fig. 2 (a)) is much broader than the grating resonance (due to the low impedance contrast, as will be discussed further later in the paper). When the grating resonance and the slit resonance overlap in spectrum at certain specific periods, the two resonance modes couple to each other and produce the familiar anti-crossing behavior. The resonant peak which is red-shifted with increasing period is actually the lower branch of the anti-crossing. The higher branch is coupled to a higher order grating resonance so that it is not observable due to the finite numerical aperture used in our experiment. The interference between a broad resonance (the slit resonance) and a sharp resonance (the grating resonance) gives rise to the typical Fano-type line shape, as confirmed by the asymmetric shape of the resonant peak in the measured transmission or the dip in reflection. The transmission vs. wavelength presented in this $2 \mathrm{D}$ color map for the period used in the experiment agrees with the measurement data (see the Discussion section and the Supplementary information online). At the period of $500 \mathrm{~nm}$, the grating resonance overlaps the central wavelength of the slit resonance so that a strong coupling is formed. Due to the high-Q characteristics of the grating resonance ${ }^{3,4}$, the coupled slit/grating resonance is much sharper than the intrinsic slit resonance, making it attractive to the spectrum filter application. With decreasing period, the grating resonance gradually moves away from the slit resonance towards shorter wavelengths. Consequently, the slit and grating resonance 
(a)

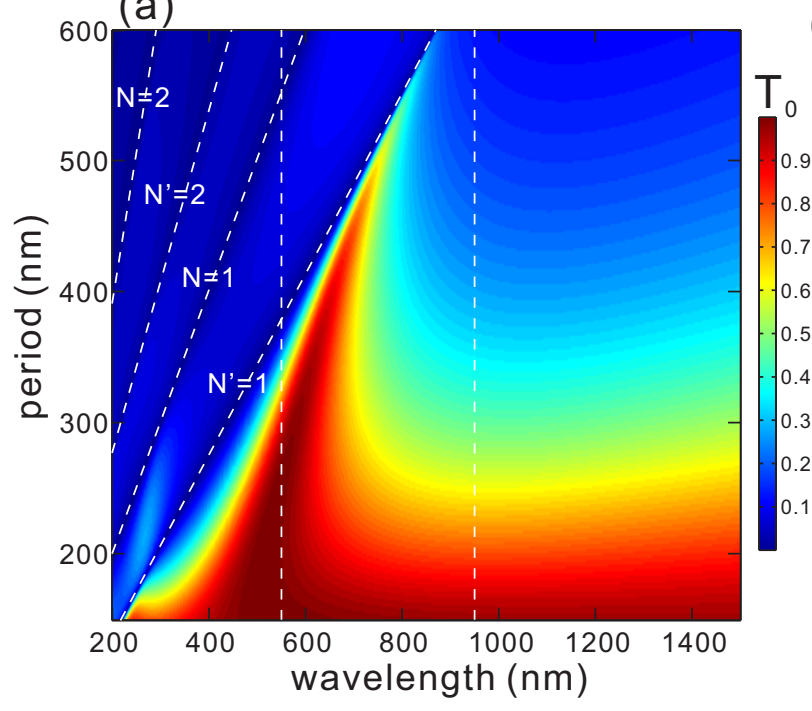

$\mathrm{p} 500 \mathrm{p} 400 \quad \mathrm{p} 300 \quad \mathrm{p} 200$

(b)

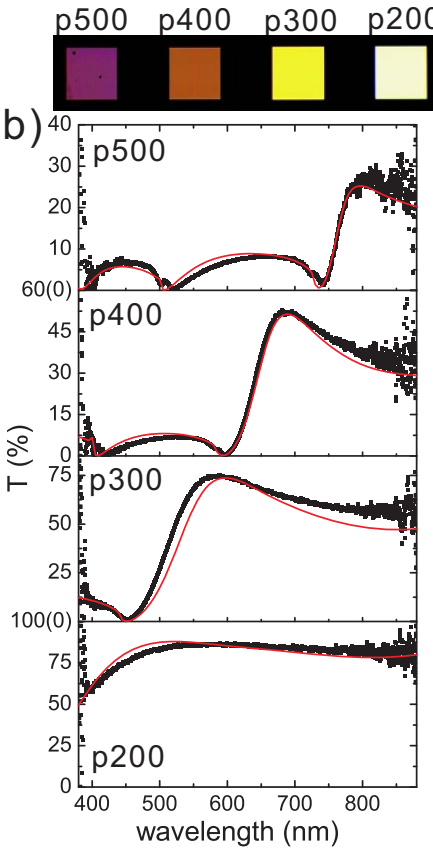

p500 p400 p300 p200

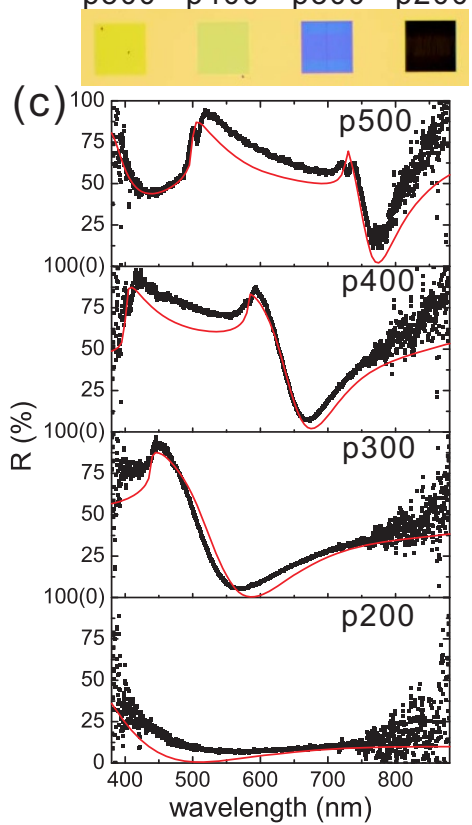

Figure $2 \mid$ Transmission and reflection measurements on the slit arrays with four different periods, in comparison with a period dependent transmission spectra based on the theoretical model. (a) shows the 2D plot of a period dependent zeroth order transmission spectra based on the theoretical model. The oblique dashed lines mark the grating resonances of different orders. The band between the two vertical dashed lines marks out the slit resonance. (b) and (c) show the measured transmission and reflection spectra (black dots) together with RCWA simulations (red curves) of the fused silica supported Aluminum slit arrays at four different periods $(200 \mathrm{~nm}, 300 \mathrm{~nm}, 400 \mathrm{~nm}$ and $500 \mathrm{~nm})$. Slit width $(\sim 110 \mathrm{~nm})$ and slit height $(\sim 170 \mathrm{~nm})$ remain invariant for different periods. Insets show the transmission and the reflection photographs of the four samples. Each square is $200 \mu \mathrm{m} \times 200 \mu \mathrm{m}$.

become separated, resulting in a broad transmission peak due to the slit resonance and a sharp transmission dip due to grating resonance. Decoupling of the two resonances with decreasing period is also manifested by the reduced angle dependence as shown in Fig. 3. As well known, the grating resonance is strongly dependent on the incident angle. As shown in Fig. 3, with an increasing incident angle, each grating resonance splits into two branches due to the phase matching requirement. The branch shifting to shorter wavelengths represents the forward diffracted light whose $k_{x}$ has the same sign as that of the incident light. The branch shifting to longer wavelengths represents the backward diffracted light whose $k_{x}$ has the opposite sign to that of the incident light. On the other hand, the slit resonance is intrinsically independent of angle ${ }^{17}$. At the period of $400 \mathrm{~nm}$, the backward branch from the first order grating resonance at the grating/substrate interface (marked as $b^{\prime}$ ) meets the slit resonance, forming a strong coupling between the two. The hybrid mode takes on the characteristics of both, e.g. the overall transmission peak becomes angle-dependent as in the case of grating resonance. The theoretical plot with an extended angle range (0-20 deg) (Fig. 3 (c)) shows that the transmission peak shifts to longer wavelengths with increasing angle. When the period decreases to $300 \mathrm{~nm}$, the $b^{\prime}$ diffraction branch is farther away from the slit resonance so that the two resonances decouple and consequently the transmission peak due to the slit resonance becomes less angle-dependent. At the period of $300 \mathrm{~nm}$, an angle-insensitive range from 0 to $8 \mathrm{deg}$ is observed in the theoretical plot in Fig. 3 (d). The angular dependence behavior will be further discussed in the last part of the Results section.

The intrinsic slit resonance. From the period dependent spectra (Fig. 2) and the angle dependent spectra (Fig. 3), the slit resonance is almost decoupled from the grating resonance at the periods below $300 \mathrm{~nm}$. In this case, the transmission peak is mostly dominated by the slit resonance. In order to reveal the intrinsic slit resonance, we fabricated the metal slit arrays with very large periods to emulate the case of a single-slit. The measured transmission at such large periods (e.g. $5 \mu \mathrm{m}$ for Fig. 4 (c)) is very weak, however a broad peak in transmission accompanied by a broad dip in reflection are still discernable (Fig. 4 (d)). The oscillatory data superimposed on the broad background is well above the noise level in the measurement. Although the zeroth order transmission is experimentally accessible, it is not a good representation of the slit resonance, especially at a large period because many diffraction orders of light transmitting through the slit cannot be collected by the finite aperture in the experiment. Therefore, we introduce the slit power enhancement factor, which is the ratio of the power flow inside slits $\left(\left\langle S_{\text {slit }}\right\rangle\right)$ to that of the incident light $\left(\left\langle S_{i}\right\rangle\right)$. At normal incidence, it can be expressed as

$$
\frac{\left\langle\mathrm{S}_{\text {slit }}\right\rangle}{\left\langle\mathrm{S}_{i}\right\rangle}=\left|a^{-}\right|^{2}-\left|a^{+}\right|^{2} .
$$

The light power inside the slits is directly influenced by slit resonances so that the slit power enhancement factor is more sensitive to slit dimension itself than to grating structure. In experiment, even though the light power inside the slits spreads into different diffraction orders, the slit power enhancement factor encompass all the diffraction orders and therefore remains high at large periods. By plotting this quantity versus wavelength and period in a $2 \mathrm{D}$ contour map, the slit resonances are clearly revealed against the grating resonances at all periods, as shown in Fig. 4 (b). The two vertical stripes in the $2 \mathrm{D}$ contour map of $\left\langle S_{\text {slit }}\right\rangle /\left\langle S_{i}\right\rangle$ (Fig. 4 (b)) are due to the first and the second order Fabry-Perot resonance in a single slit, whose bandwidths can be read out from Fig. 4 (a) as $\sim 450 \mathrm{~nm}$ and $\sim 100 \mathrm{~nm}$, respectively, and they are obviously independent of period; whereas the dense array of oblique lines are due to the grating resonances, and are linearly dependent on the period as expected. The slit resonance does cut through a number of grating resonances as shown in Fig. 4(b), forming the coupled hybrid modes as discussed above, which are responsible for the small oscillations superposed 

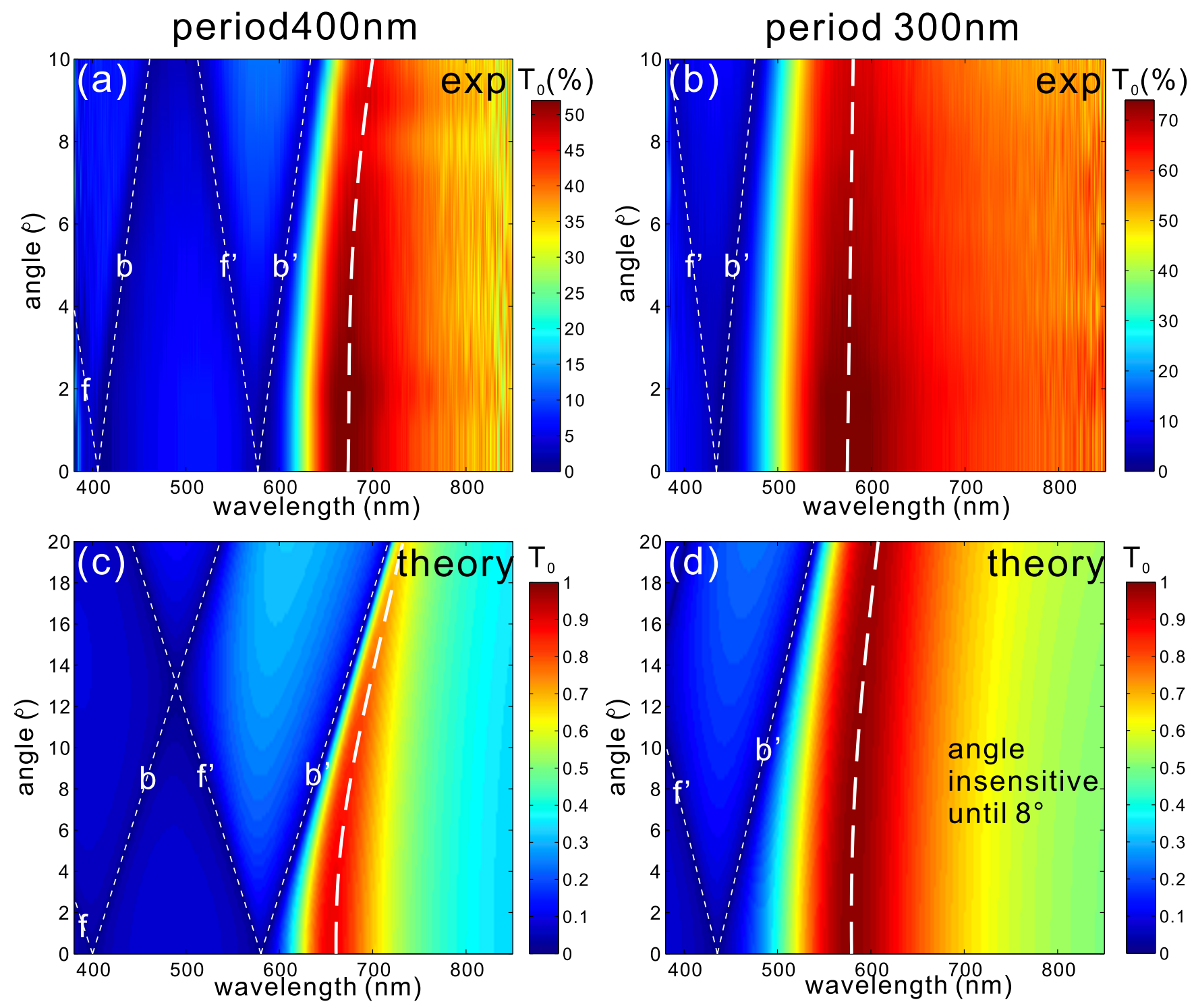

Figure 3 Angle-dependent transmission measurement at two periods together with the theoretical results. (a) and (b) show the measured angledependent transmission spectra for fused silica supported Aluminum slit arrays with a period of $400 \mathrm{~nm}$ and a period of $300 \mathrm{~nm}$, respectively. The slit width of the structure is $\sim 110 \mathrm{~nm}$ and the slit height is $\sim 170 \mathrm{~nm}$. (c) and (d) show the angle-dependent transmission spectra by the theoretical model in an extend angle range. The branches marked out by the thin dashed lines are due to grating resonances. $f$ and $b$ mark the forward and the backward branches, respectively, of the grating resonance at the grating/air interface; $f$ and $b^{\prime}$ the forward and the backward branches at the grating/substrate interface. The thick dashed lines marks the transmission peak.

over the broad slit resonance in the measured spectrum (black squares in Fig. 4 (c) and (d)), and qualitatively agree with the Rigorous Coupled Wave Analysis (RCWA) simulation results (red curves in Fig. 4(c) and 4(d), also see the Discussion section).

Dimished slit resonance with further decreasing period---transition to broadband polarizer. An illuminated metal slit with a deep sub-wavelength dimension can be considered as a dipole $e^{37-39}$. When driven by a TM wave with the direction of the electric field crossing the slit, polarization charges are accumulated at the corners and consequently distort the nearby field. At either the entrance or the exit side of the slits the incident or the outgoing plane waves should couple into or out of the waveguide modes in the slits, which creates an impedance mismatch at the two interfaces. The impedance difference leads to partial light reflection from the two surfaces. Such impedance mismatch and the constructive field buildup of slit waveguide modes establishes a Fabry-Perot type resonance-this is the nature of the slit resonance discussed above.
In the case of an array of slits, the separation between each slit plays an important role in deciding the impedance mismatch and consequently the light confinement. For a $400 \mathrm{~nm}$ period, as shown in Fig. 5 (a) p400, the induced dipoles are separated from each other by sufficient distance. Thus, the light field around each dipole is distorted, leading to a large impedance mismatch between the incident plane wave and the slit waveguide mode. Thus, light is strongly reflected at both ends of the slit, which is desirable for forming high-Q F-P resonance modes. When the period decreases to $200 \mathrm{~nm}$ while the slit width remains $110 \mathrm{~nm}$, the induced dipoles are adjacent to each other end to end as shown in Fig. 5 (a) p200 so that the accumulated charges tend to cancel out. Thus, the light field is only slightly distorted. Therefore the impedance mismatch to air is significantly reduced, leading to a much weaker F-P resonance and significantly broadened transmission "peak". Indeed the broadband polarizer is achieved when the slit resonance becomes very weak so that high transmission is maintained across a wide wavelength range of the slit resonance. 
(a)
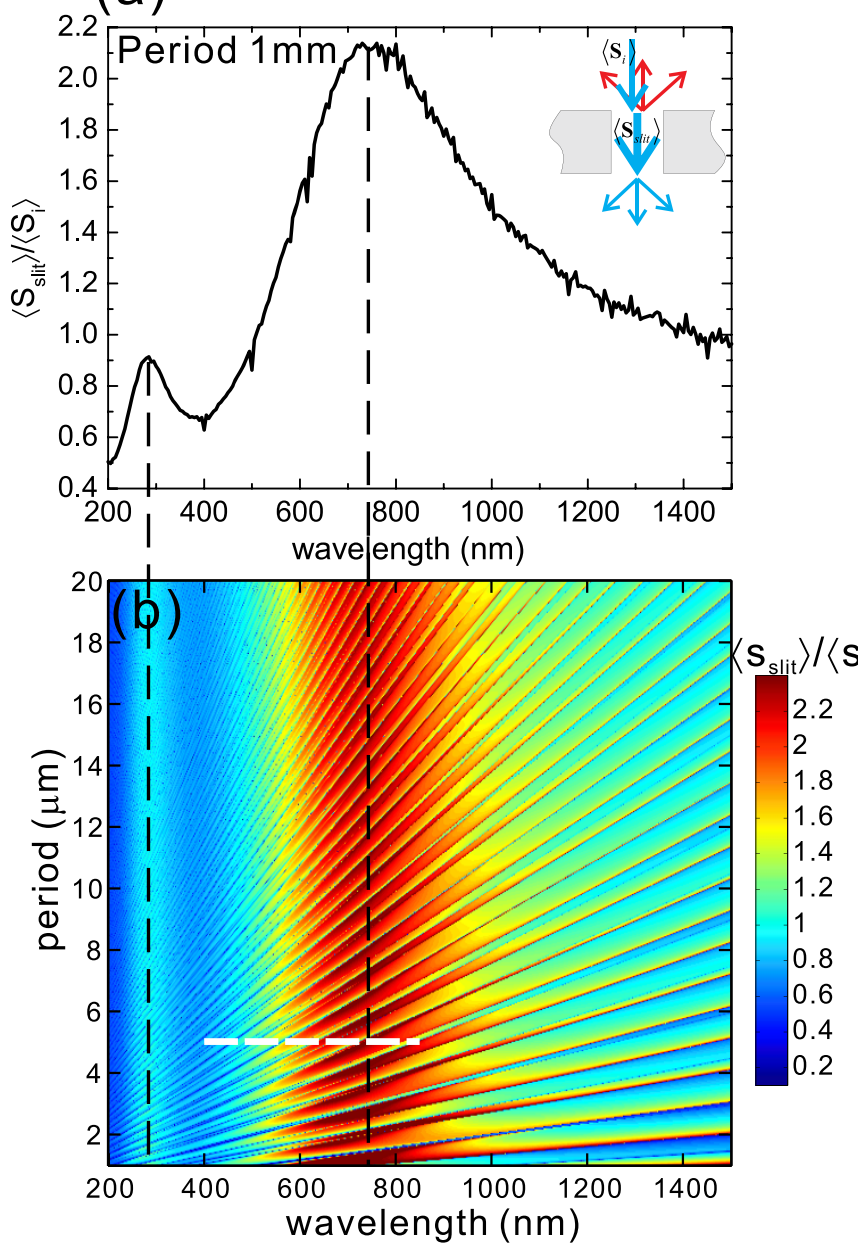

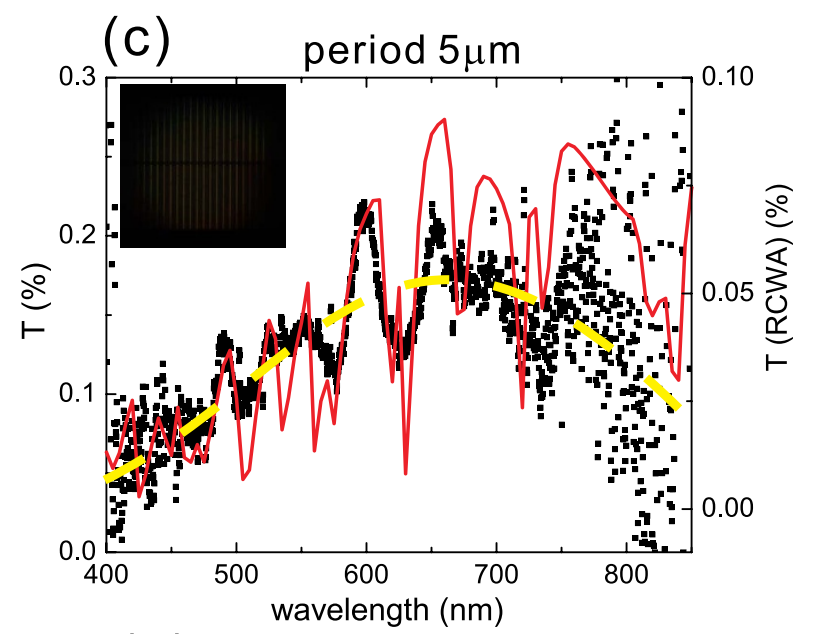

(d)

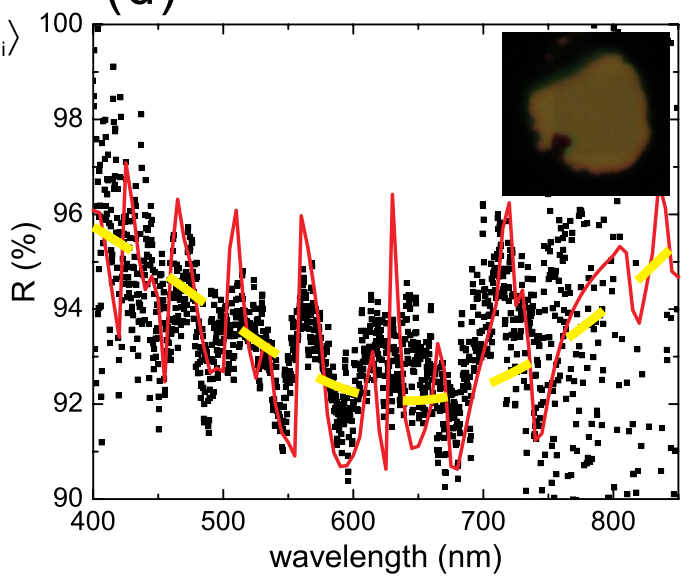

Figure $4 \mid$ Identifying the intrinsic slit resonance. (a) Spectrum plot of the slit power enhancement factor $\left(\left\langle S_{\text {slit }}\right\rangle /\left\langle S_{i}\right\rangle\right)$ at the period of $1 \mathrm{~mm}$ based on the theoretical model. Inset illustrates the definition of the slit power enhancement factor as a ratio of the power flow inside the slit to that of the incident light. (b) Period-dependent spectra of the slit power enhancement factor. The white dashed line marks out the spectrum at the period of $5 \mu \mathrm{m}$ to compare with the measured data. (c) and (d) Measured transmission and reflection spectra (black dots) together with RCWA simulations (red lines) of the fused silica supported Aluminum slit array with a period of $5 \mu \mathrm{m}$. An eye-guide line (yellow dashed) is plotted in (c) and (d) to reveal the broad slit resonance. The slit width is $\sim 110 \mathrm{~nm}$ and the slit height is $\sim 170 \mathrm{~nm}$. The reflection spectrum is in agreement with the simulation while the transmission is about three times higher. It is probably due to the high magnification of the microscope objective $(40 \times)$ that was used in transmission measurement in order to enhance the signal by collecting several diffraction orders instead of only the zeroth order. Insets show the transmission and the reflection photographs of the slit array.

Finally we provide an analytical approach to describe the above mechanism. The metal film with perforating slit array can be mapped into a dielectric slab with an effective refractive index $\left(n_{\text {eff }}\right)$ and an effective thickness $\left(h_{e f f}\right)$ by comparing either the reflection coefficient or the transmission coefficient of a free-standing metal film with that of the dielectric slab. In this sense, the slit resonance of the metal film with perforating slit array corresponds to the F-P resonance of the effective dielectric slab. This method follows that prescribed in Ref. 33. Assuming the metal to be a perfect conductor, $n_{\text {eff }}$ and $h_{\text {eff }}$ are expressed as

$$
n_{\text {eff }}=\frac{1}{\sum_{m=-\infty}^{+\infty} \frac{a}{d} \operatorname{sinc}\left(m \pi \frac{a}{d}\right) \frac{1}{\sqrt{1-\left(m \frac{\lambda}{d}\right)^{2}}}}
$$

and $h_{\text {eff }}=h / n_{\text {eff. }}$ Through this analogy, the impedance mismatch is

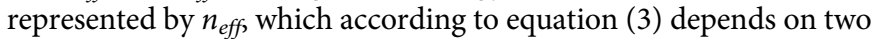
ratios, the ratio of slit width to period $(a / d)$ and the ratio of wavelength to period $(\lambda / d)$. In the long wavelength limit (i.e. $\lambda / d \rightarrow \infty)$, the higher orders in the sum of equation (3) only introduce a small imaginary correction, and then $n_{\text {eff }}$ approximately equals to $d / a$, which is proportional to period. Consequently, with decreasing period the reflectance reduces as shown in Fig. 5 (d). It is well known that the reflectance of a film determines the finesse of the F-P etalon made of this film. With reducing reflectance, the F-P resonance of the effective dielectric slab (i.e. the slit resonance of a metal slit array) diminishes so that the transmission become broadband and flat. At smaller wavelengths, although $n_{\text {eff }}$ becomes a complex number as shown by two examples $(600 \mathrm{~nm}$ and $1000 \mathrm{~nm})$ in Fig. 5 (c), the corresponding reflectance at the metal slit array still decreases with decreasing period (Fig. $5(\mathrm{~d})$ ), which confirms the diminishing process of the slit resonance.

Angular dependence. As the metal slit arrays transition from a spectrum filter to a polarizer due to decoupling of the slit/grating resonance, the angular dependence reduces simultaneously. Thus, a polarizer made of the metal slit array structure becomes increasingly angle-independent with reducing periods. On the other hand, an angle-insensitive spectrum filter is desirable in many applications. As discussed, the angular dependence can be reduced by decreasing the period. However the transmission peak broadens significantly 
(a)
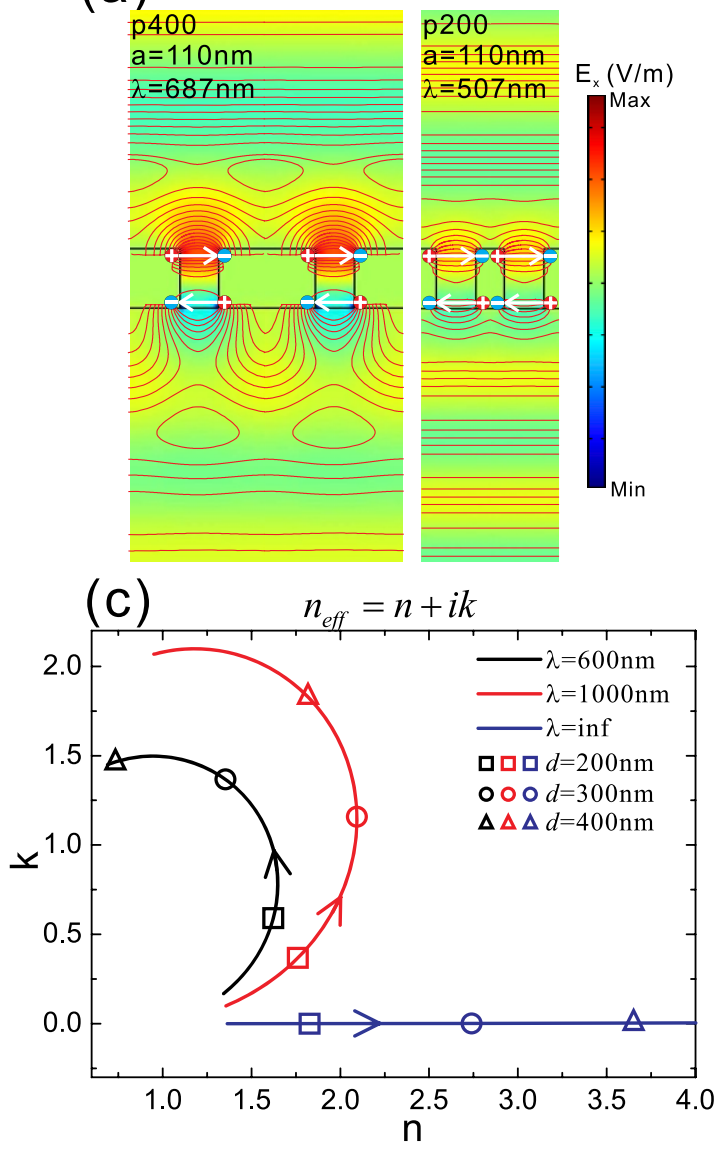

(b)
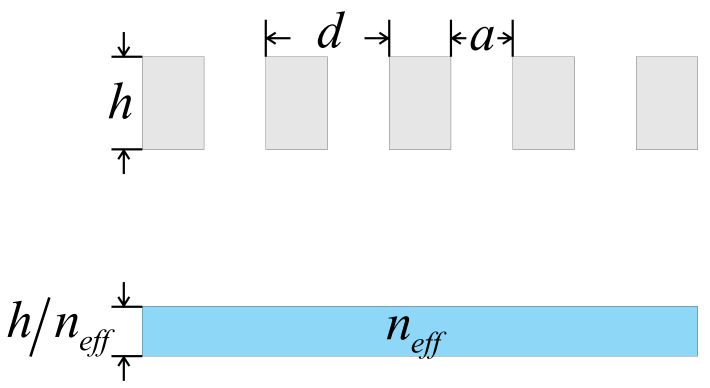

(d)

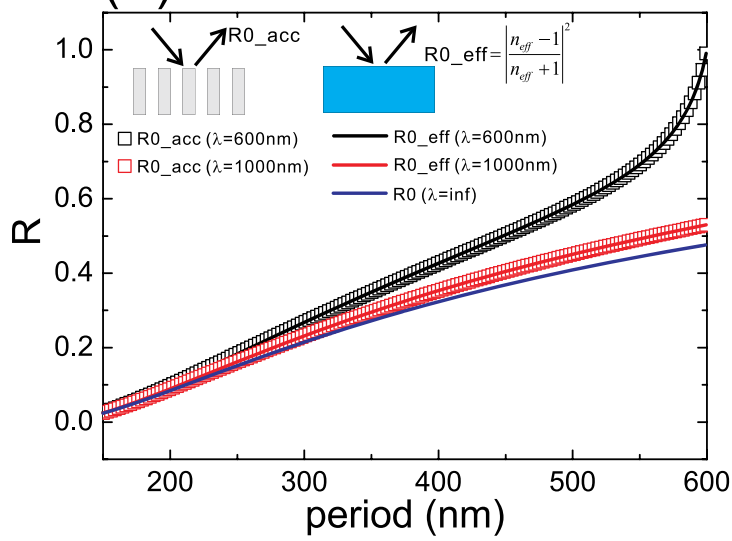

Figure 5 Illustration of the slit resonance diminishing with period decreasing. (a) Simulated $x$-component E field distributions and electric field lines of two metal slit arrays at their own resonant wavelengths, i.e. the period of $400 \mathrm{~nm}$ at its resonant wavelength of $687 \mathrm{~nm}$; the period of $200 \mathrm{~nm}$ at its resonant wavelength of $507 \mathrm{~nm}$. As revealed by the calculated electric lines, the light field near the slit openings in the $400 \mathrm{~nm}$-period array is distorted more severely than that in the $200 \mathrm{~nm}$-period array. Accordingly, the calculated $x$-component of electric field $\left(E_{x}\right)$ reveals a stronger on-resonance light field inside the slits of the $400 \mathrm{~nm}$ period than that of the $200 \mathrm{~nm}$ period. The max value of the colorbar is $2.44 \times 10^{5} \mathrm{~V} / \mathrm{m}$; the min value is $-2.90 \times$ $10^{5} \mathrm{~V} / \mathrm{m}$. (b) Sketch of the analogy between a metal slit array layer and a dielectric slab. (c) Evolution of the effective refractive index $\left(n_{e f f}\right)$ with period at three different wavelengths (i.e. $600 \mathrm{~nm}, 1000 \mathrm{~nm}$ and infinite) plotted in a complex plane with the horizontal and vertical axis to be the real and imaginary part of $n_{\text {eff. }}$ Period increases along each curve in the direction of the arrows. Three periods, i.e. $200 \mathrm{~nm}, 300 \mathrm{~nm}$ and $400 \mathrm{~nm}$ are marked out by squares, circles and triangles. (d) Evolution of the reflectance from the metal slit array with period. The accurate reflectance (R0_acc) is in squares and the reflectance calculated through the effective index (R0_eff) is in solid lines. At an infinitely large wavelength, these two quantities are identical. The expression of R0_acc can be found in the Supplementary information online.

due to two reasons: 1) the decoupling of the sharp grating resonance from the broad intrinsic slit resonance increases the band width of the transmission peak. 2) Reduction in the impedance mismatch between the light mode inside and outside the slits further broadens the slit resonance. Therefore, in order to design an angular insensitive spectrum filter using this structure, the bandwidth of the slit resonance should be reduced. This can be done by either shrinking the slit width or filling the slit with a high index dielectric, so that the $\mathrm{Q}$ factor of the slit cavity is enhanced and resonance bandwidth reduced.

\section{Discussion}

Our theoretical model assumes the metal to be a perfect conductor, which is an approximation of the real metal used in the experiments. The validity of this approximation in describing the transition from a spectrum filter to a polarizer in a real metal slit array is studied. As a direct comparison, the transmission spectra predicted by the theoretical model, the measurement results and the simulation by using RCWA based on real metal index are plotted together in Fig. 6 (a). The period of the metal slit array is $400 \mathrm{~nm}$. The slit width and the slit height are $110 \mathrm{~nm}$ and $170 \mathrm{~nm}$, respectively. Clearly the theoretical model based on ideal metal and RCWA simulation based on real metal both reproduce the main features observed in the experiment, i.e. the grating resonances (represented by the two dips) and the slit resonance (represented by the broad peak). There are also discrepancies in the transmission amplitudes and resonance peak positions: 1) the spectrum of the $\mathrm{Al}$ slit array (either the measurement or the RCWA simulation) shows a lower transmission peak at the slit resonance than the theoretical prediction based on ideal metal, which is due to the optical loss in $\mathrm{Al} ; 2$ ) both the slit resonance and the grating resonances are red-shifted in the spectrum of the Al slit array with respect to the theoretical model. This can be explained by the different types of propagating waves for the real metal and the ideal metal case, in particular the difference in propagation constants. Simply put it, the grating resonance (Fig. 6 (b)) in the ideal metal case is due to the diffracted wave along the surface direction having free space momentum, while for real metal it is due to the coupling to the surface plasmon polariton (SPP) wave, which is known to have higher momentum due to its special dispersion, and therefore occurs at longer wavelength (see Supplementary Fig. S3 (c) online). 
(a)

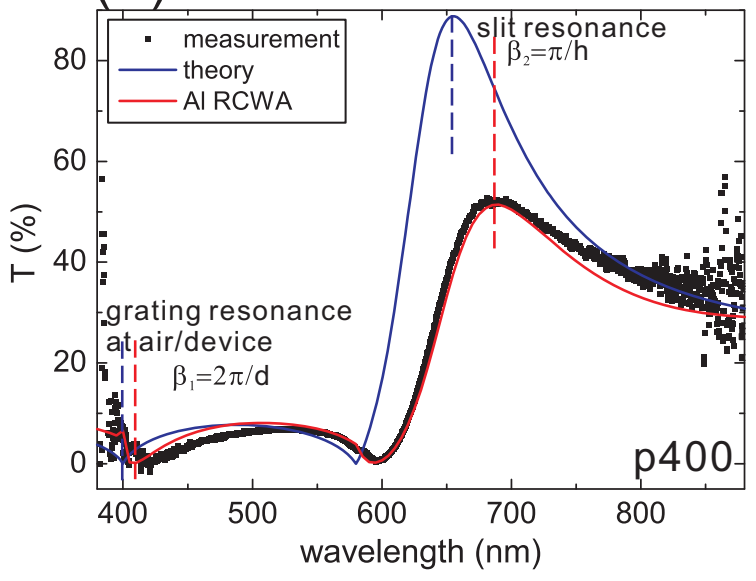

(b)

\section{grating resonance}

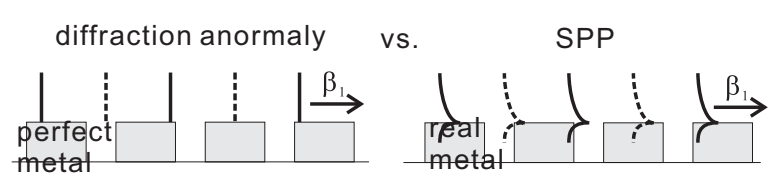

(c)

slit resonance

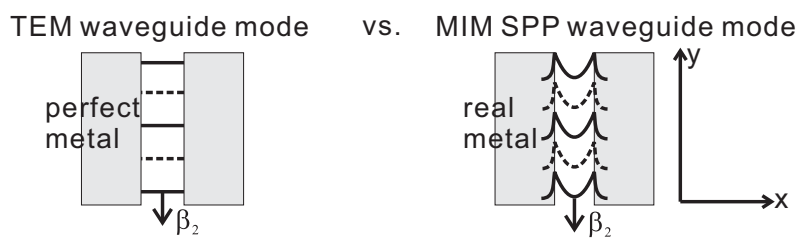

Figure 6 Comparison between a slit array made of a perfect metal and that made of a real metal. (a) Transmittance of a slit array made from a perfect metal (theoretical prediction: blue curve) or Al (measured data: black dots; RCWA simulation: red curve). The period is $400 \mathrm{~nm}$, the slit width $110 \mathrm{~nm}$ and the slit height $170 \mathrm{~nm}$. (b) Sketch of light diffraction along the surface of a perfect metal slit array versus the SPP wave at a real metal slit array. (c) Sketch of the TEM waveguide mode in a perfect metal slit versus the MIM SPP mode in a real metal slit.

Similarly, the slit resonance (Fig. 6 (c)) with the ideal metal walls is due to the interference of TEM wave with free space momentum; while that for real metal ( $\mathrm{Al})$ the resonance is due to coupled SPP modes inside the metal slits, again having higher momentum than free space photons and therefore occurs at longer wavelength. This effect can be seen more clearly with Ag metal, which is a better plasmonic metal in the visible band. The detailed discussion can be found in the Supplementary information online, along with the calculated dispersion of various cases described above.

Since the theoretical model is based on the perfect metal approximation, all the analysis becomes more accurate at longer wavelengths where real metals approach to ideal metals. Therefore, this work provides some guidelines in designing the spectrum filters and polarizers not only in the visible-NIR range, but also in the IR and microwave bands as well.

In conclusion, the transition from a spectrum filter (resonant transmission) to a polarizer (broadband transmission) based on the metal slit array structure can be tuned by the period of the array. The distinct transmission peak in the former case is due to a coupled slit/grating resonance, which broadens with decreasing period as the coupled two resonances become separated, and the slit resonance which is intrinsically much broader than the grating resonance dominates the transmission peak. As the slit resonance diminishes due to reduced light confinement, a broadband transmission (a polarizer behavior) is reached. Another effect of decreasing period is that the transmission peak becomes less angle sensitive due to decoupling of the grating resonance (angular dependent) from the slit resonance (angular independent). Therefore, the polarizer is naturally angle insensitive due to the very small period, while a spectrum filter can be made angle insensitive by increasing the light confinement in each slit to fight against the broadening of the transmission peak with decreasing period.

\section{Methods}

Fabrication of the nano-slit arrays. The nano-slit arrays with smaller periods (from $200 \mathrm{~nm}$ to $500 \mathrm{~nm}$ ) are created through standard e-beam lithography and reactive ion etching. The slit array with a large period $(5 \mu \mathrm{m})$ is created by focused ion beam. Both processes were performed on a Aluminum film (170 nm thick) deposited on a fused silica substrate. The slit height remains at $\sim 170 \mathrm{~nm}$ as all slits perforate the $\mathrm{Al}$ film. The slit width was kept at $\sim 110 \mathrm{~nm}$.

Transmission and reflection measurements. The transmission measurement was done by using an invert microscope (Nikon Eclipse TE300) equipped with a spectrometer (Ocean Optics HR4000CG-UV-NIR). The illuminating light from a halogen light bulb is collimated before impinging on the sample. On the other side, the transmitting light is collected by an objective lens with a magnification of $40 \times$ and a numerical aperture (N.A.) of 0.75 . Since the grating periods (from $200 \mathrm{~nm}$ to 500 $\mathrm{nm}$ ) we studied are smaller than most part of the wavelength range (from $380 \mathrm{~nm}$ to $880 \mathrm{~nm}$ ), the objective lens only collects the zeroth order transmission. The reflection measurement was performed on the same platform. In this case, a low-magnification $(4 \times)$ objective lens is used to transmit the illuminating light and to collect the reflection light. The small N.A. (0.1) of the objective lens ensures little beam divergence. The angle-dependent transmission measurement was conducted with a small tilting stage which can be fit into the sampling space of the invert microscope. The measured angle range $\left(0-10^{\circ}\right)$ is limited by the tilting stage.

Simulations. The transmission or reflection spectra shown in red lines in Fig. 2 (b) and (c) and Fig. 4 (c) and (d) were simulated by RCWA. The field distribution of $E_{x}$ as shown in Fig. 5 (a) was simulated by finite element method with the commercial software COMSOL Multiphysics. For both methods, the optical constant of Al was taken from Ref. 40 and that of fused silica from Ref. 41 .

1. Porto, J. A., García-Vidal, F. J. \& Pendry, J. B. Transmission Resonances on Metallic Gratings with Very Narrow Slits. Phys. Rev. Lett. 83, 2845-2848 (1999).

2. Lalanne, P., Hugonin, J. P., Astilean, S., Palamaru, M. \& Möller, K. D. One-mode model and Airy-like formulae for one-dimensional metallic gratings. J. Opt. A 2, 48-51 (2000).

3. Collin, S., Pardo, F., Teissier, R. \& Pelouard, J.-L. Strong discontinuities in the complex photonic band structure of transmission metallic gratings. Phys. Rev. B 63, 033107 (2001).

4. Collin, S., Pardo, F., Teissier, R. \& Pelouard, J.-L. Horizontal and vertical surface resonances in transmission metallic gratings. J. Opt. A 4, S154-S160 (2002).

5. García-Vidal, F. J. \& Martin-Moreno, L. Transmission and focusing of light in one-dimensional periodically nanostructured metals. Phys. Rev. B 66, 155412 (2002).

6. Liu, W. C. \& Tsai, D. P. Optical tunneling effect of surface plasmon polaritons and localized surface plasmon resonance. Phys. Rev. B 65, 155423 (2002).

7. Hooper, I. R. \& Sambles, J. R. Surface plasmon polaritons on thin-slab metal gratings. Phys. Rev. B 67, 235404 (2003).

8. Lee, K. G. \& Park, Q. H. Coupling of Surface Plasmon Polaritons and Light in Metallic Nanoslits. Phys. Rev. Lett. 95, 103902 (2005).

9. Marquier, F., Greffet, J.-J., Collin, S., Pardo, F. \& Pelouard, J. L. Resonant transmission through a metallic film due to coupled modes. Opt. Express 13, 70-76 (2005).

10. Catrysse, P. B., Veronis, G., Shin, H., Shen, J.-T. \& Fan, S. Guided modes supported by plasmonic films with a periodic arrangement of subwavelength slits. Appl. Phys. Lett. 88, 031101 (2006).

11. García-Vidal, F. J., Martin-Moreno, L., Ebbesen, T. W. \& Kuipers, L. Light passing through subwavelength apertures. Rev. Mod. Phys. 82, 729-787 (2010).

12. Roszkiewicz, A. \& Nasalski, W. Reflection suppression and absorption enhancement of optical field at thin metal gratings with narrow slits. Opt. Lett. 37, 3759-3761 (2012).

13. Kim, T. J., Thio, T., Ebbesen, T. W., Grupp, D. E. \& Lezec, H. J. Control of optical transmission through metals perforated with subwavelength hole arrays. Opt. Lett. 24, 256-258 (1999).

14. Xu, T. et al. Structural Colors: from Plasmonic to Carbon Nanostructures. Small 7, 3128-3136 (2011). 
15. Yu, Z., Veronis, G., Fan, S. \& Brongersma, M. L. Design of midinfrared photodetectors enhanced by surface plasmons on grating structures. Appl. Phys. Lett. 89, 151116 (2006)

16. Kaplan, A. F., Xu, T. \& Guo, L. J. High efficiency resonance-based spectrum filters with tunable transmission bandwidth fabricated using nanoimprint lithography. Appl. Phys. Lett. 99, 143111 (2011).

17. Wu, Y.-K. R., Hollowell, A. E., Zhang, C. \& Guo, L. J. Angle-Insensitive Structural Colours based on Metallic Nanocavities and Coloured Pixels beyond the Diffraction Limit. Sci. Rep. 3, 1194 (2013).

18. Wang, J. J. et al. High-performance nanowire-grid polarizers. Opt. Lett. 30, 195-197 (2005).

19. Chen, L., Wang, J. J., Walters, F., Deng, X. \& Buonanno, M. Large flexible nanowire grid visible polarizer made by nanoimprint lithography. Appl. Phys. Lett. 90, 063111 (2007).

20. Wang, J. J., Walters, F., Liu, X., Sciortino, P. \& Deng, X. Appl. Phys. Lett. 90, 061104 (2007).

21. Pelletier, V. et al. Aluminum Nanowire Polarizing Grids: Fabrication and Analysis. Proc. of SPIE 6462, 646217-1 (2007).

22. Shin, Y. J., Pina-Hernandez, C., Wu, Y.-K., Ok, J. G. \& Guo, L. J. Facile route of flexible wire grid polarizer fabrication by angled-evaporations of aluminum on two sidewalls of an imprinted nanograting. Nanotechnology 23, 344018 (2012).

23. Hollowell, A. E. \& Guo, L. J. Nanowire Grid Polarizers Integrated into Flexible, Gas Permeable, Biocompatible Materials and Contact Lenses. Adv. Opt. Mater. 1, 343-348 (2013).

24. Ebbesen, T. W., Lezec, H. J., Ghaemi, H. F., Thio, T. \& Wolff, P. A. Extraordinary optical transmission through sub-wavelength hole arrays. Nature 391, 667-669 (1998).

25. Schröter, U. \& Heitmann, D. Surface-plasmon-enhanced transmission through metallic gratings. Phys. Rev. B 58, 15419 (1998).

26. Lochbihler, H. \& Depine, R. Highly conducting wire gratings in the resonance region. Appl. Opt. 32, 3459-3465 (1993).

27. Lochbihler, H. Surface polaritons on gold-wire gratings. Phys. Rev. B $\mathbf{5 0}$ 4795-4801 (1994).

28. Astilean, S., Lalanne, P. \& Palamaru, M. Light transmission through metallic channels much smaller than the wavelength. Opt. Commun. 175, 265-273 (2000).

29. Sobnack, M. B., Tan, W. C., Wanstall, N. P., Preist, T. W. \& Sambles, J. R. Stationary Surface Plasmons on a Zero-Order Metal Grating. Phys. Rev. Lett. 80, 5667-5670 (1998).

30. López-Rios, T., Mendoza, D., García-Vidal, F. J., Sánchez-Dehesa, J. \& Pannetier, B. Surface Shape Resonances in Lamellar Metallic Gratings. Phys. Rev. Lett. 81, 665-668 (1998).

31. Perchec, J. L., Quémerais, P., Barbara, A. \& López-Ríos, T. Why Metallic Surfaces with Grooves a Few Nanometers Deep and Wide May Strongly Absorb Visible Light. Phys. Rev. Lett. 100, 066408 (2008).

32. Pardo, F., Bouchon, P., Haïdar, R. \& Pelouard, J.-L. Light Funneling Mechanism Explained by Magnetoelectric Interference. Phys. Rev. Lett. 107, 093902 (2011).
33. Shen, J. T., Catrysse, P. B. \& Fan, S. Mechanism for Designing Metallic Metamaterials with a High Index of Refraction. Phys. Rev. Lett. 94, 197401 (2005).

34. Botten, L. C., Craig, M. S. \& McPhedran, R. C. Highly Conducting Lamellar Diffraction Gratings. Optica Acta 28, 1103-1106 (1981).

35. Botten, L. C., Craig, M. S., McPhedran, R. C., Adams, J. L. \& Andrewartha, J. R. The Finitely Conducting Lamellar Diffraction Grating. Optica Acta 28 1087-1102 (1981).

36. Sheng, P., Stepleman, R. S. \& Sanda, P. N. Exact eigenfunctions for square-wave gratings: Application to diffraction and surface-plasrnon calculations. Phys. Rev. B 26, 2907-2916 (1982).

37. Lalanne, P. \& Hugonin, J. P. Interaction between optical nano-objects at metallodielectric interface. Nature 2, 551-556 (2006).

38. Lalanne, P., Hugonin, J. P., Liu, H. T. \& Wang, B. A microscopic view of the electromagnetic properties of sub-l metallic surfaces. Surf. Sci. Rep. 64, 453-469 (2009).

39. Nikitin, A. Yu., García-Vidal, F. J. \& Martín-Moreno, L. Surface Electromagnetic Field Radiated by a Subwavelength Hole in a Metal Film. Phys. Rev. Lett. 105 073902 (2010).

40. Rakic, A. D. Algorithm for the determination of intrinsic optical constants of metal films: application to aluminum. Appl. Opt. 34, 4755 (1995).

41. Bass, M. et al. Handbook of Optics, Third Edition Volume IV: Optical Properties of Materials, Nonlinear Optics, Quantum Optics (McGraw-Hill Professional, 2009).

\section{Acknowledgments}

The authors would like to acknowledge the support by AFOSR (Dr. Harold Weinstock) and NSF ECCS.

\section{Author contributions}

L.J.G. and J.Z. conceived the idea. J.Z. performed the experiments and developed the theoretical model. J.Z. and L.J.G. discussed the results and wrote the article. L.J.G. supervised the work

\section{Additional information}

Supplementary information accompanies this paper at http://www.nature.com/ scientificreports

Competing financial interests: The authors declare no competing financial interests.

How to cite this article: Zhou, J. \& Guo, L.J. Transition from a spectrum filter to a polarizer in a metallic nano-slit array. Sci. Rep. 4, 3614; DOI:10.1038/srep03614 (2014).

(c) (i) (2) This work is licensed under a Creative Commons Attribution-

BY NG SA NonCommercial-ShareAlike 3.0 Unported license. To view a copy of this license, visit http://creativecommons.org/licenses/by-nc-sa/3.0 